\title{
Avaliação de Estimadores de Canais Clássicos em Sistemas Cooperativos
}

\author{
Leonardo Zavareze da Costa, Dimas Irion Alves, Renato Machado e Nelson Jorge Schuch.
}

\begin{abstract}
Resumo-Este artigo apresenta uma avaliação de diferentes estimadores de canais clássicos aplicados em dois protocolos de comunicação cooperativa. Os protocolos considerados neste trabalho são o Amplifica e Encaminha (AF - Amplify-Forward) e o Decodifica e Encaminha Seletivo (SDF - Select Decode-andForward) e os algoritmos avaliados são o MMSE, LMS e RMS. Os resultados de simulações são apresentados na forma BER versus SNR. Baseados nos resultados de simulação, faz-se uma análise de custo-benefício dos três algoritmos de estimação de canal em função da complexidade de implementação e desempenho obtido.
\end{abstract}

\section{Palavras-Chave-MMSE, LMS, RMS, AF, DF.}

Abstract-The paper presents an evaluation of different classical channel estimators used in two cooperative communication protocols. The protocols considered in this work are the Amplify-and-Forward (AF) and Select Decode-Forward (SDF), and the algorithms evaluated are the MMSE, LMS and RMS. Simulations results are presented in terms of BER versus SNR. Based on the simulation results, a trade-off evaluation between implementation complexity and performance for the three channel estimation algorithms is considered.

Keywords-MMSE, LMS, RMS, AF, DF.

\section{INTRODUÇÃO}

Os sistemas cooperativos têm atraído um crescente interesse na área de telecomunicações devido às novas possibilidades de se explorar a diversidade espacial cooperativa para a transmissão de dados. Considerando uma rede de nós, é possível criar uma rede MIMO (Multiple-Input Multiple-Output) virtual e explorar os múltiplos pontos de enlace, espalhados geograficamente, permitindo a obtenção de diversidade espacial (também chamada de diversidade cooperativa), antes somente alcançada em sistemas com múltiplas antenas [1].

Nos pontos de enlace intermediários entre o transmissor e receptor, chamados relays, o sinal enviado pode sofrer apenas uma amplificação, ou passar por algum tipo de processamento, dependendo do protocolo utilizado. Os protocolos utilizados neste trabalho são o Amplifica e Encaminha (AF - Amplifyand-Forward) e o Decodifica e Encaminha Seletivo (SDF - Selected Decode-and-Forward). No protocolo AF, o sinal recebido pelo relay é amplificado e retransmitido ao nó destino, enquanto que no SDF o nó relay considera ou não a decodificação do sinal antes de encaminha-la ao nó destino. Se

Leonardo Z. da Costa, Dimas I. Alves, Renato Machado e Nelson J. Schuch, Centro Regional Sul de Pesquisas Espaciais - CRS/INPEMCTI, em colaboração com o Laboratório de Ciências Espaciais de Santa Maria - LACESM/CT/UFSM, Santa Maria-RS, Brasil. E-mails: leonardozavareze@gmail.com, dimasirion977@gmail.com, renatomachado@ieee.org,njschuch@lacesm.ufsm.br o canal fonte-relay está acima de um determinado threshold, então o sinal é decodificado e encaminhado para o nó destino, caso contrário o nó relay ignora a transmissão do nó fonte. Essa estratégia aumenta a confiabilidade da comunicação [2].

Para poder se detectar um sinal no receptor é necessário se conhecer os valores dos coeficientes do canal de comunicação. Esses coeficientes podem ser estimados por algoritmos conhecidos como estimadores de canais. Os estimadores MMSE (Minimum Mean Square Estimation), LMS (Last-mean Square) e RLS (Recursive Least Square) são considerados neste artigo. O trabalho apresenta os resultados das simulações em termos de taxa de erro de bit (BER - Bit Error Rate) versus razão sinal-ruído (SNR - Signal-to-Noise Ratio). Com os resultados de simulação, faz-se uma comparação dos três algoritmos de estimação de canal em termos de complexidade e desempenho.

\section{Modelo do Sistema}

O modelo utilizado é apresentado na Fig. 1. No primeiro slot de tempo, a fonte envia sinais para o destino e para o relay. Se o protocolo aplicado for o AF, o relay amplifica o sinal e o reenvia ao destino. Se for o SDF, há uma verificação dos dados recebidos (threshold) antes de decodificá-lo e re-enviá-lo. Assume-se que os canais estão sujeitos a um desvanecimento do tipo Rayleigh.

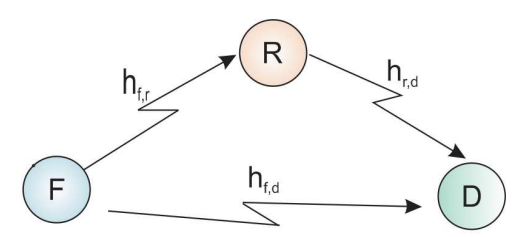

Fig. 1. Modelo de sistema.

Os sinais recebidos pelo destino e relay, no primeiro slot de tempo, podem ser descritos como:

$$
\begin{aligned}
& y_{f, r}=\sqrt{P_{f}} x h_{f, r}+n_{f, r}, \\
& y_{f, d}=\sqrt{P_{f}} x h_{f, d}+n_{f, d},
\end{aligned}
$$

em que, $P$ é a potência de envio, $x$ é o símbolo enviado, $h$ é o valor do coeficiente do canal e $n$ é o ruído Gaussiano branco, modelado como variável aleatória complexa de média zero e variância $N_{0}$. No segundo slot de tempo, o sinal recebido pelo destino, considerando o protocolo AF, é definido como:

$$
y_{r, d}=\sqrt{\frac{P}{P\left|h_{s, r}\right|^{2}+N_{0}}} y_{f, r} h_{r, d}+n_{r, d}
$$


em que, $y_{r, d}$ é o sinal recebido pelo destino e o termo multiplicativo de $y_{f, r}$ é conhecido como fator de amplificação.

Considerando o protocolo SDF, o sinal recebido pelo destino, no segundo slot de tempo, é definido por:

$$
y_{r, d}=\sqrt{\hat{P}_{2}} x h_{r, d}+\eta_{r, d}
$$

em que

$$
\hat{P}_{2}=\left\{\begin{aligned}
P_{2}, & \text { se } P\left|h_{f, r}\right|^{2} \geq \text { threshold }, \\
0, & \text { se } P\left|h_{f, r}\right|^{2}<\text { threshold } .
\end{aligned}\right.
$$

O processo que ocorre no destino, na chegada dos sinais enviados pelo relay e pela fonte, é a combinação dos sinais $y_{s d}$ e $y_{r d}$ a partir do combinador de máxima verossimilhança.

\section{ESTIMADORES}

Para este trabalho, os seguintes estimadores foram utilizados:

- MMSE - Minimum Mean Square Error: Objetiva encontrar valores para parâmetros que apresentem o menor erro quadrático médio (MSE). Pode-se definir o algoritmo do estimador como sendo:

$$
h_{M M S E}=y\left(p^{H} R_{H} p+\sigma_{n}^{2} I\right)^{-1} p^{H} R_{H}
$$

em que $h$ é o coeficiente do canal estimado, $p$ são os símbolos de treinamento e $R$ é a matriz de correlação espacial [3].

- LMS - Least Mean Square: Versão estocástica do método do gradiente, que se aproxima da solução de Wiener e baseia-se em minimizar o MSE [4]. Pode-se definir o algoritmo do estimador como sendo:

$$
h(n+1)=h(n)+p(n)\left[d^{*}(n)-p^{h} h(n)\right]
$$

em que $d$ é o sinal recebido no receptor.

- RLS - Recursive Least Square: Método similar ao LMS, porém mais complexo, visto que realiza a inversão da matriz de autocorrelação [4]. Pode-se definir o algoritmo do estimador como sendo:

$$
\begin{gathered}
g(n)=\frac{\lambda^{-1} R^{-1}(N-1) p(n)}{1+\lambda^{-1} p^{H}(n) R^{-1}(n-1) p(n)} \\
h(n+1)=h(n)+g(n) e^{*}(n) \\
R_{D}^{-1}=\lambda^{-1}\left[1-g(n) p^{H}\right] R^{-1}(n-1)
\end{gathered}
$$

em que, $g$ é o vetor de ganho, $\lambda$ é o fator de esquecimento e $e$ é o vetor de erro de estimação. Baseando-se nas equações apresentadas, é possível notar que os algoritmos LMS e RLS são iterativos.

\section{Resultados}

Para as simulações, considera-se que os sinais são mapeados em uma constelação QPSK. Os resultados das simulações são expressos na forma de BER $\times$ SNR. Realiza-se a transmissão de $10^{8}$ símbolos, sendo que, cada frame foi composto por 250 bits com $10 \%$ de bits de treinamento. Para simulações com SDF, utilizou-se um valor de threshold igual a 8. A Fig. 2 mostra o desempenho de BER obtido com protocolos Amplifica e Encaminha (AFF) e Decodifica

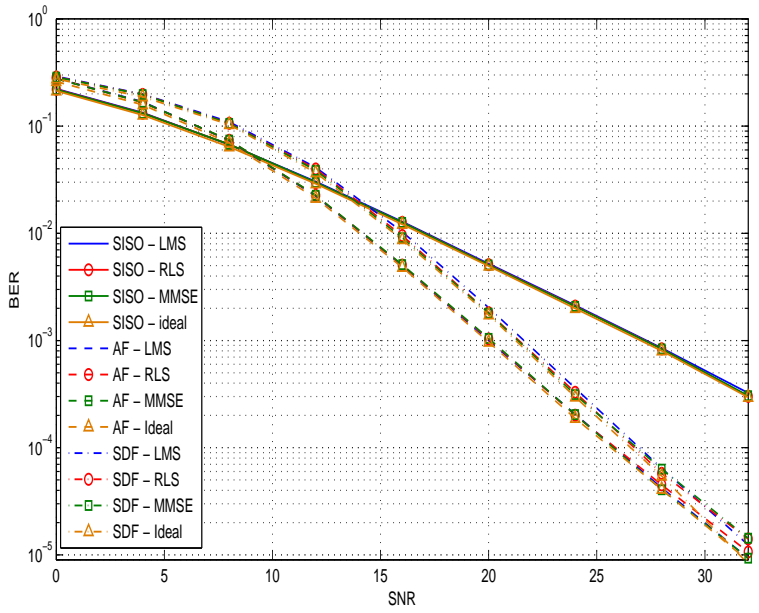

Fig. 2. Análise de BER para protocolos AF e SDF utilizando os estimadores MMSE, LMS e RLS.

e Encaminha Seletivo (SDF). Para o protocolo AmplificaEncaminha, nota-se uma diferença menor entre desempenho dos estimadores, sendo que o LMS provê um desempenho um pouco melhor que os outros algoritmos analisados. Para o protocolo SDF, percebe-se um pouco mais a diferença entre o desempenho dos estimadores. Entretanto, os estimadores fornecem um desempenho muito semelhante em termos de $\mathrm{BER}$, considerando que o modelo do sistema não envolve um canal de comunicação com mobilidade.

\section{CONCLusões E COMEnTÁRIOS Finais}

Para o modelo de sistema adotado, as simulações comprovaram a diferença entre o sistema convencional SISO e os modelos MIMO virtuais. Comprova-se que, utilizando diferentes protocolos no relay, pode-se obter um aumento da derivada BER. Apesar da diferença de complexidade dos algoritmos considerados neste trabalho, o desempenho de BER observado é praticamente o mesmo. O resultado provavelmente ocorreu, pois, neste primeiro estudo, considerou-se apenas canais não variantes no tempo, o que impede uma comparação mais detalhada entre os algoritmos. Futuramente, pretendese inserir o efeito Doppler no modelo do sistema e realizar novas simulações para se avaliar o comportamento destes estimadores sob o efeito de mobilidade relativa entre os nós do sistema.

\section{REFERÊNCIAS}

[1] J. N. Laneman and G. W. Wornell, "Distributed space-time coded protocols for exploiting cooperative diversity in wireless networks,' IEEE Trans. Inf. Theory, vol. 49, no. 10, pp. 2415-2425, Oct. 2003.

[2] K.J. Ray Liu, A. K. Sadek, W. Su e A. Kwasinski, Cooperative Communications and Networking. Cambridge University Press , 2009.

[3] M. Biguesh, A. B. Gershman, Training-Based MIMO Channel Estimation: A Study of Estimator Tradeoffs and Optimal Training Signals. IEEE Transactions on Signal Processing, Vol 54, No.3, Março de 2006.

[4] A. Goldsmith, Wireless Communications. Stanford University, 2004. 